\title{
Optical Characteristics of CdSSe Films Prepared by Thermal Evaporation Technique
}

\author{
Zaidunn T. Mohammed Noori* \\ Received 14, March, 2009 \\ Accepted 14, February, 2010
}

\begin{abstract}
:
Thin films of cadmium sulphoselenide (CdSSe) have been prepared by a thermal evaporation method on glass substrate, and with pressure of $4 \times 10^{-5}$ mbar. The optical constants such as (refractive index $\mathrm{n}$, dielectric constant $\varepsilon_{\mathrm{i}, \mathrm{r}}$ and Extinction coefficient $\kappa)$ of the deposition films were obtained from the analysis of the experimental recorded transmittance spectral data. The optical band gap of (CdSSe) films is calculate from $(\alpha h v)^{2}$ vs. photon energy curve. CdSSe films have a direct energy gap, and the values of the energy gap were found to increase when increasing annealing temperature. The band gap of the films varies from $1.68-2.39 \mathrm{eV}$.
\end{abstract}

Key words: Optical energy gap, annealing of Thin films of cadmium sulphoselenide,

\section{Introduction:}

Cadmium sulphoselenide

(CdSSe) is well known as a II-VI compound semiconductor suitable for solar energy conversion with a photovoltaic cell because the band gap, i.e. $2.28 \mathrm{eV}$, is fit to the spectrum of sunlight. Several methods of the film processing such as vacuum evaporation, chemical vapor deposition (CVD), chemical bath deposition, spray pyrolysis, electro deposition etc have been employed for the deposition of thin CdSe films[1-5]. In this work, the thermal evaporation technique was successfully employed for the deposition of CdSSe films. The II-VI ternary semiconducting compounds, belonging to the cadmium chalcogenide family $(\mathrm{CdS}, \mathrm{CdTe}$, CdSe), are considered to be very important materials for a wide spectrum of optoelectronic applications as having specific physical properties such as direct band-gap widths, high absorption coefficients in the visible and infrared part of the solar spectrum, good electrical properties (e.g. carrier mobility and lifetime) and increased capability in obtaining adjustable n- or p-type conductivity by doping. Several techniques were employed for the growth of thin films of the ternary CdSSe. In this work, $\mathrm{CdS}_{0.5} \mathrm{Se}_{1-x}$ films were deposited by the thermal evaporation technique at different annealing temperature and the results obtained on these films are presented and discussed. particular, CdSSe is also a very good laser media and can be used to produce lasing in the visible spectral region

\section{Material and Method:}

Cadmium sulphoselenide thin films were deposited on glass substrates using the thermal evaporation technique, The films were synthesized at room temperature. The glass substrates were cleaned at first by a mild soap lution, then in boiling water and ultrasonic cleaner and finally degreased in alcohol vapor. The

*Optics Technology Department -Dijllah University College 
chamber was evacuated by conventional rotary and diffusion pump combination to a base pressure of $4 \times 10^{-5}$ mbar. The thickness of the films was determined using Fizeau fringe method. Transmission measurements were performed with an accuracy of $3 \%$ in the spectral range from 400 to $850 \mathrm{~nm}$ using UV spectrophotometer.

\section{X-Ray Diffraction Procedur:}

Philips vertical powder diffractometer type PW1050 was used. Spectra were obtained by using $\mathrm{Ni}$ filtered Cuk $\alpha$ radiation $\lambda=0.15418 \mathrm{~nm}$ operated at $40 \mathrm{kV}$ and $20 \mathrm{~mA}$. The scan range $2 \theta$ was chosen between 20-50 degrees run at a scan rate of $0.2 \mathrm{deg} / \mathrm{s}$.

\section{Results and Discussion:}

Fig.1.a Shows X-Ray diffraction patterns of $\mathrm{CdS}{ }_{0.5} \mathrm{Se}_{1-x}$ powder the complete amorphous structure. The XRay diffraction of annealed films at $\left(\mathrm{T}_{\mathrm{a}}=323 \& 348 \mathrm{~K}\right)$ are shown in Fig.1.b and $c$. These films have amorphous structure for all samples at $\left(\mathrm{T}_{\mathrm{a}}=303\right.$ (as deposited) $\& 348 \mathrm{~K}$ ) but the films becomes crystalline when annealed at $\left(\mathrm{T}_{\mathrm{a}}=323 \quad \& 348 \mathrm{~K}\right)$ for two hours as shown in Figs.1a-c .It should be noted that the X-ray diffraction spectra exhibits two weak broad humps at $\mathrm{T}_{\mathrm{a}}=323 \mathrm{~K} \& \mathrm{~T}_{\mathrm{a}}=348 \mathrm{~K}$ at Bragg angles $(2 \theta=24.3$ and 29.1) respectively, which undoubtedly indicate that these films become polycrystalline

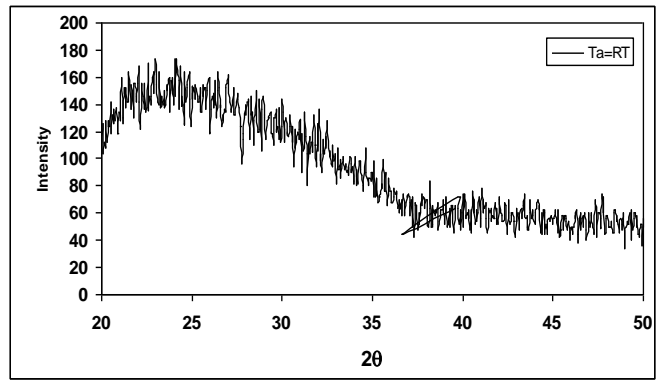

(a)

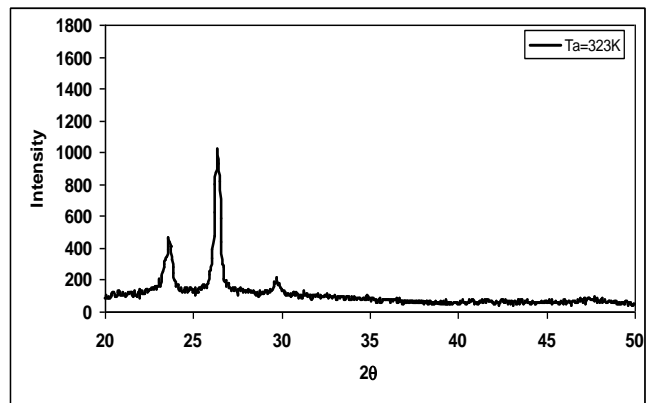

(b)

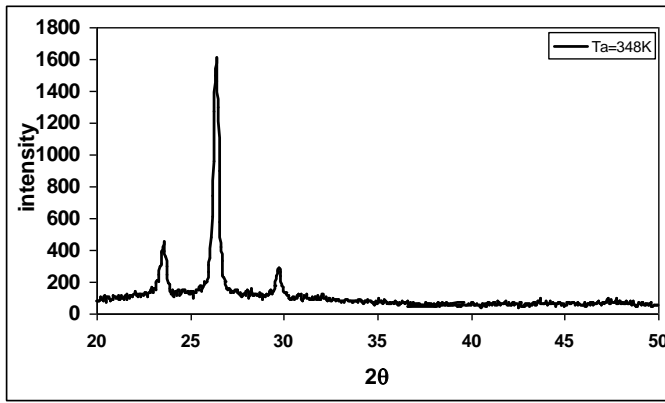

(c)

Fig.(1) shows the XRD patterns of the CdSSe thin films deposited at different annealing temperature : aRT , b-Ta=323K , $c-T a=348 k$

Fig.2. Shows the optical transmission spectra of CdSSe thin films from this figure, in general, it can be observed that the peak of transmittance spectra of CdSSe films shift toward the shorter wavelengths with increasing the annealing temperatures. This shifting is due the structural improvement that is represented by increasing the crystallites size; the values of transmission are nearly in agreement with 


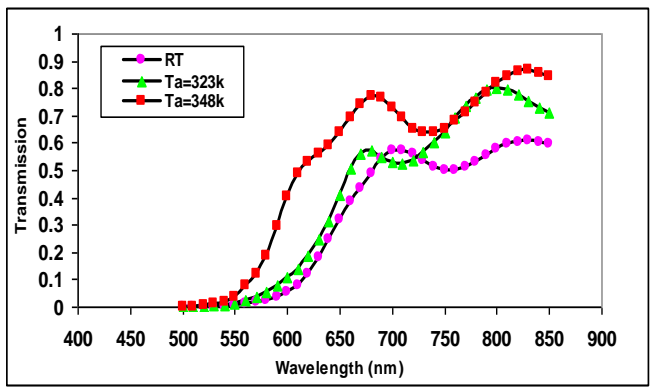

Fig.2. The transmission spectra for CdSSe films

The absorption spectra for CdSSe thin films, having different annealing temperature, are shown in fig.(2).This has opposite behavior of the transmittance spectra[6]. It is observed from this Fig.(3) that the absorbance decrease with increasing annealing temperature, this may be due to the increase in the transmittance of the related films this result agree with the result of studied by Anlian et al..[7]

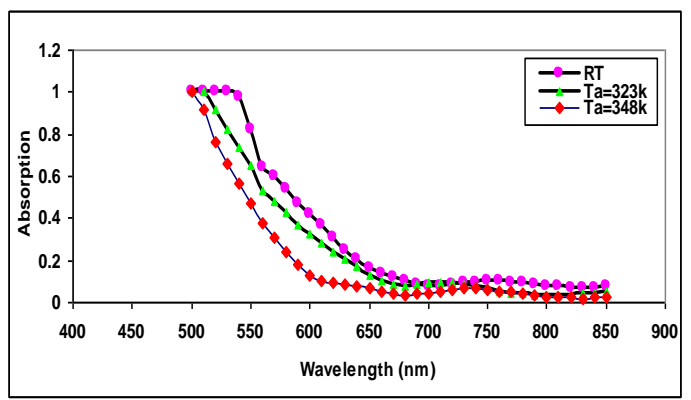

Fig. 3. The absorbance spectra for CdSSe films

The absorption coefficient of CdSSe films for the as prepared and annealed films are illustrated in fig.(4).

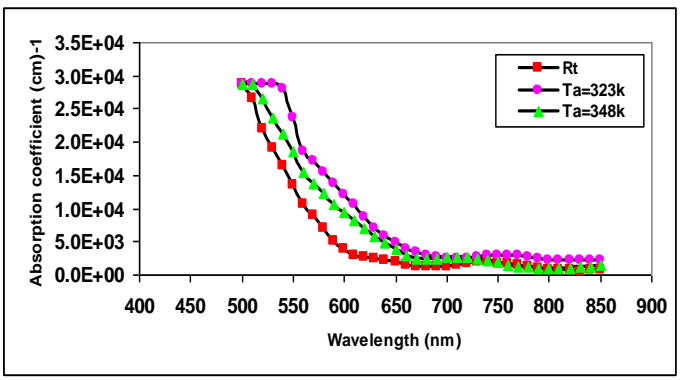

Fig.4. The absorption coefficient for CdSSe films

From this fig.(4) the absorption coefficient of CdSSe films are characterized by strong absorption at short wavelength region within the range $(500-850) \mathrm{nm}$. We can observe that from this figure the absorption coefficient decrease with the increasing of wavelength [7], the absorption coefficient $(\alpha)$ of CdSSe films was calculated using lambert law

$\operatorname{Ln}\left(\frac{I_{O}}{I}\right)=2.303 \mathrm{~A}=\alpha \mathrm{d} \ldots \ldots .(1)$

Where $I_{0}$ and $I$ are the incident and the transmitted light respectivel.

A is the optical absorbance and (d) is the film thickness.

Fig. 5(a-c) shows the locally amplified optical microscope (OM) image of the ternary CdSSe films, which indicates that most of the films are straight with a uniform width of several micrometers and their length can be extended to more than several hundreds of micrometers Morphology and surface roughness of CdSSe films change significantly when Increase in annealing (from RT to $348 \mathrm{~K}$ ) increases the nucleation density of the crystals rather than the growth of already nucleated ones . 


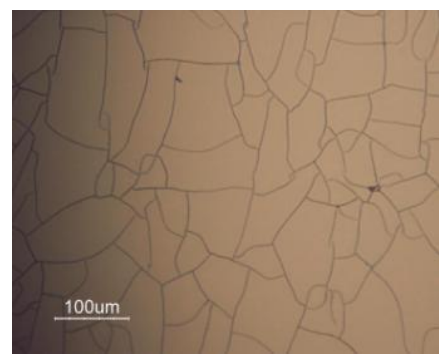

(a)

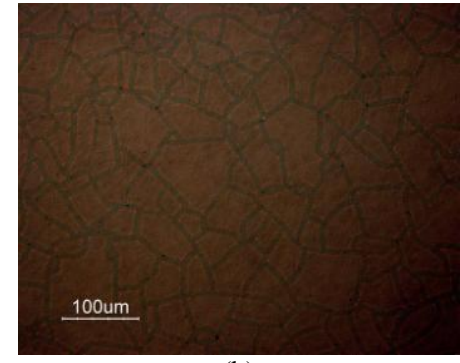

(b)

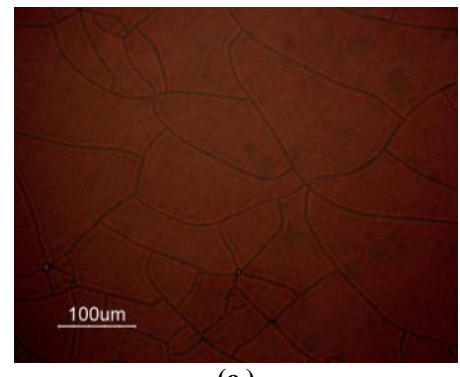

(c)

Fig. 5 Optical Microscope(OP) of the ternary CdSSe films after thermal treatment (a) for film at $\operatorname{Rt}(300 k) \quad$ (b) $\mathrm{Ta}=323 \mathrm{k}$ (c) $\mathrm{Ta}=348 \mathrm{k}$

The optical energy gap value $\left(\mathrm{E}_{g}^{\text {opt }}\right.$ ) for CdSSe films have been determined by using Touc equation, Which is used to examine type of transition by plotting $(\alpha h v)^{1 / n}$ as a function of the photon energy ho and select the optimum linear part. it is found that the first relation yields linear dependence, which describes the allowed direct transition.

To determined the possible transitions $(\alpha h v)^{1 / n} \quad$ vs. hv were plotted and corresponding band gap were obtained from extrapolating the straight portion of the graph on hv axis at $(\alpha=0)$

It is found that the first relation yields linear dependence, which describes the allowed direct transition as shown in fig.6. The direct optical energy gap for CdSSe films equal to $(1.68-2.39) \mathrm{eV}$ with increase annealing temperature from (RT to $348 \mathrm{k}$ ) this result agree with the result of studied by Murali and Venkatachalam [8].

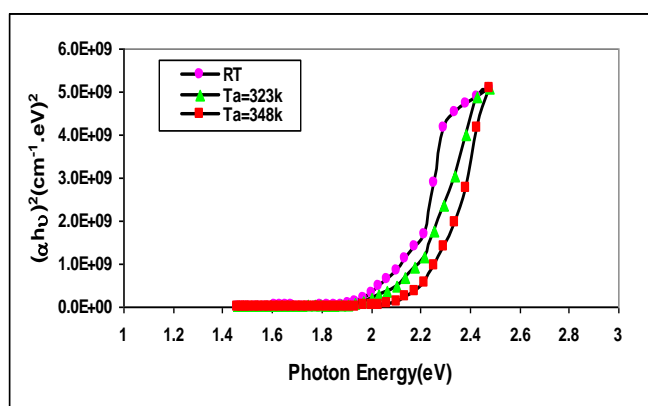

Fig.6. The plot of $(\alpha h v)^{2}$ vs. hv for CdSSe films

Fig.(7) shows the variation of refractive index with wavelength of asdeposited and annealed CdSSe. It is found from this fig.(7) that the refractive index decreases with the increasing annealing temperature

In order to determine extinction coefficient was used the the relation as follows $K=\alpha \lambda / 2 \pi$

The behavior of extinction coefficient is nearly simslar to the corresponding absorption coefficient,as shown in fig.(8) for CdSSe films at different extinction coefficient from fig.(8), we can observed that the extinction coefficient for CdSSe films increases with increasing the extinction coefficient. 


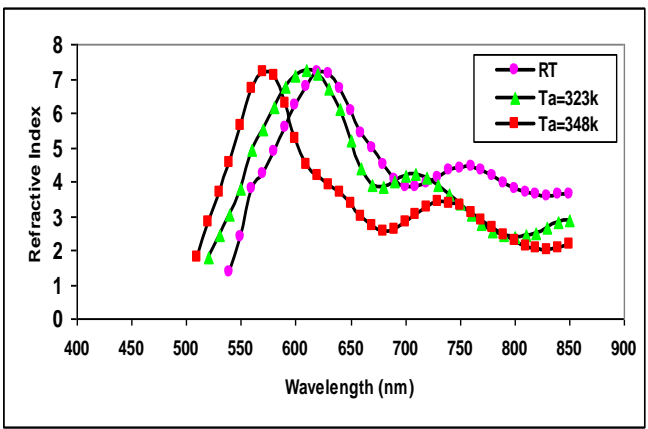

Fig.7. The Refractive index versus wavelength of incident radiation for CdSSe films

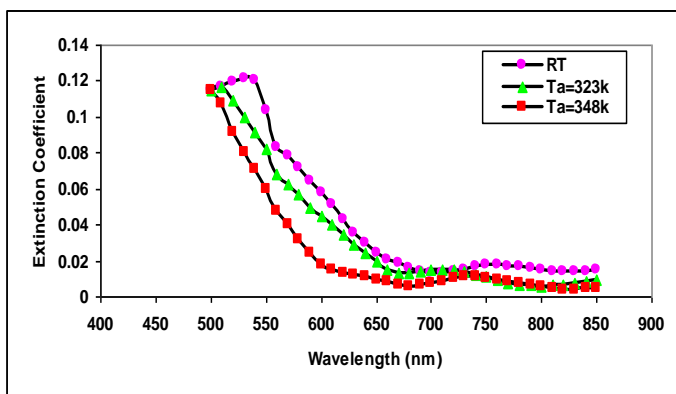

\section{Fig.8.The Extinction coefficient versus wavelength of incident radiation for CdSSe films}

The real $\varepsilon_{\mathrm{r}}$ and imaginary $\varepsilon_{\mathrm{i}}$ parts of the dielectric constant for CdSSe were determined formula

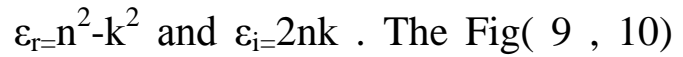
show the variation of real and imaginary part of dielectric constant respectively for $\mathrm{CdSSe}$ films respectively at different temperature. The behavior of $\epsilon_{r}$ is similar to refractive index because of the smaller value of extinction coefficient comparison to refractive index, while $\epsilon_{i}$ depends mainly on the Kvalues, which is related to the variation of absorption coefficient for CdSSe,$\epsilon_{\mathrm{r}}$ decrease with increasing annealing temperature from room temperature to $348 \stackrel{o}{k}$, while $\epsilon_{\mathrm{i}}$ increase with increasing annealing temperature the behavior is due to the change of reflectance and absorbance .

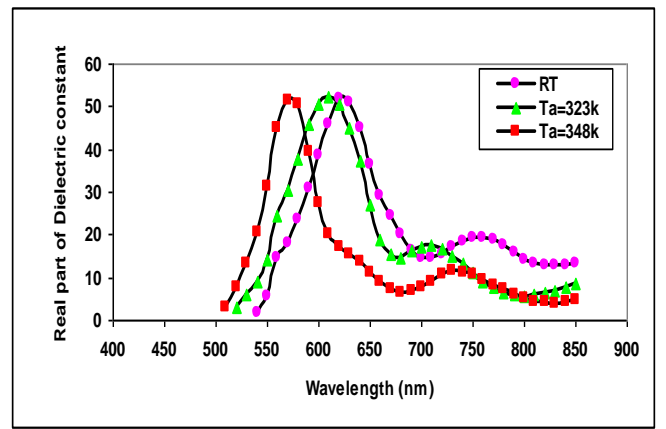

Fig.9. Real dielectric constant for CdSSe films

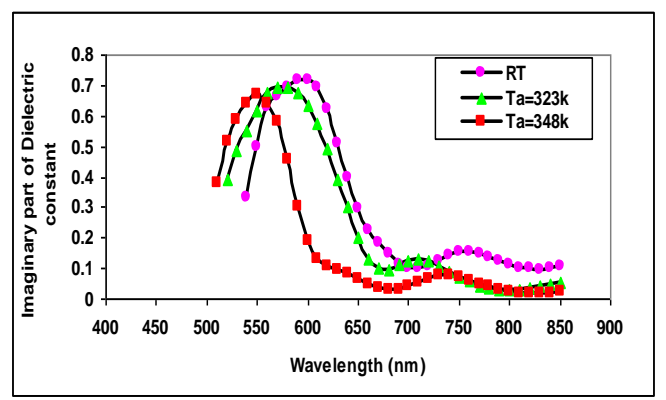

Fig.10. Imaginary dielectric constant for CdSSe films

\section{Conclusions:}

The thin films of CdSSe, deposited by thermal evaporation technique have been characterized using optical measurements and deductions to obtain such optical and solid state properties as the T-R-A spectra, optical band gap energy, extinction coefficient, refractive index, and real and imaginary dielectric constant. The variations of these with incident photon energy wavelength have been studied. All the films exhibit high transmittance $(\sim 70-80 \%)$, low reflectance in the visible near infrared region from $\sim 500 \mathrm{~nm}$ to $850 \mathrm{~nm}$, thus making the films suitable for optoelectronic devices, for instance as window layers in solar cells. The films show a direct transition.Variations in the optical constants with wavelength are found to be oscillatory in nature, which are attributed to the particular structure of the films and their thickness. 


\section{Reference:}

1. Erat, S. Metin, H. Arı, M. 2008. Materials Chemistry and Physics. 111-114.

2. Hernandez-Perez M. A., J. AguilarHernandez, G. Contreras-Puente, J. R. Vargas García, E. Rangel-Salinas Physica 2008. E: Low-dimensional Systems and Nanostructures. E40(1): 2535.

3. Ju, Z. G. Lu,Y. M. Zhang, J. Y. Wu, X. J. K. W. Liu, D. X. Zhao, Z. Z. Zhang, B. H.Li,B. Yao, D.Z. Shen, J. Cryst. Growth. 2007. Determination of the optical constants of poly crystalline $\mathrm{CdSe}$ thin film prepared by evaporaton Appl. Surf Sci, 307(1): 26 .

4. Ezema FL. And Osuji R.U 2007. Band gap shift and optical characterization of chemical bath deposition CdSSe thin films on annealing, chalcogenide letters, 4(6):69-75.

5. Bouroushian M., Karoussos D., Kosanovic T.2006. solid state Ionics. 177- 1855 .

6. Soliman L. I. and A. M. Ibrahim. 2008. Determination of optical constant of thermal evaporation CdSxSe1-x thin filme usingonl transmission spectra, Issn 1330 coden fizae 4 (12):11-16.

7. Anlian P., Liu R., Wang F., Xie S., Zou B., Zacharias M., and Wang Z. L.2006. High-Quality Alloyed CdSxSe1-x Whiskers as Waveguides with Tunable Stimulated,J. Phys. Chem. B, 110(1): 22313-22317

8. K.R.Murali and K.Venkatachalam. 2008. elecrtrical properties of sintered $\quad \mathrm{CdS}_{\mathrm{x}} \mathrm{Se}_{1-\mathrm{x},}$, Chalcogenide Letters. 5( 9): 181-186

\section{المحضرة بتقتية التبخير الحراري CdSSe الخصائص البصرية لاغشية}

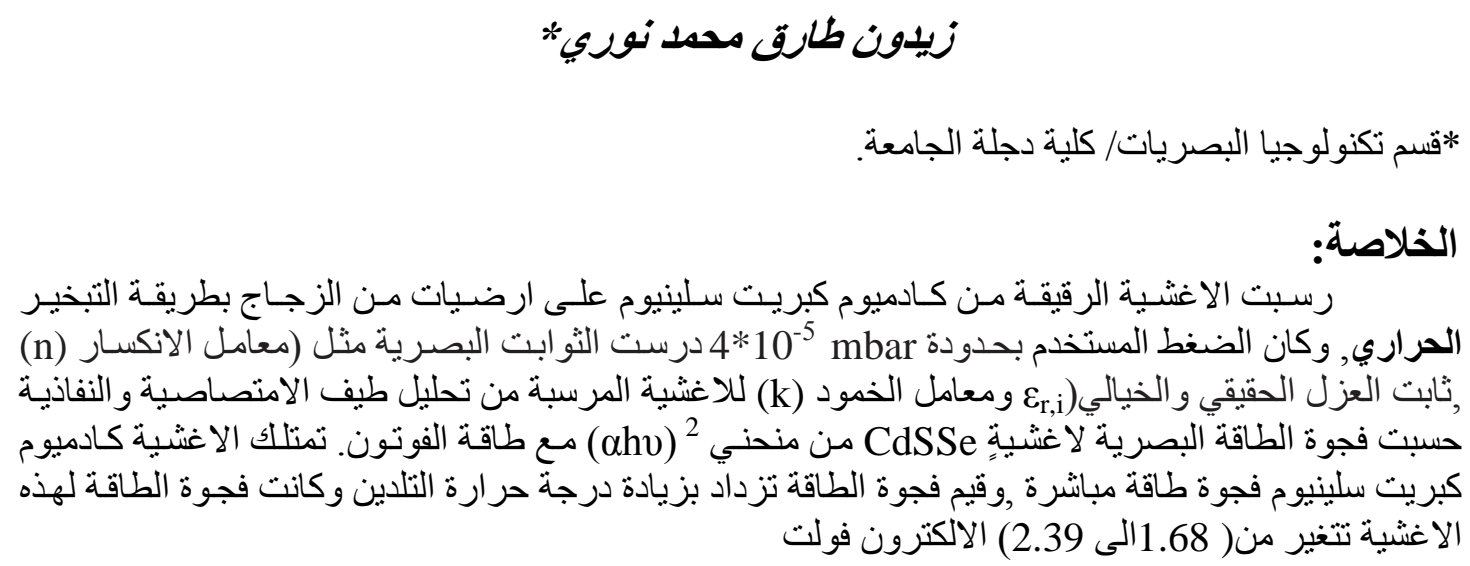

